\title{
Research Paper \\ Correlation of Social Support and Religious Orientation With Life Satisfaction in the Elderly
}

\author{
Khodamorad Momeni ${ }^{1},{ }^{*}$ Zahra Rafiee ${ }^{1}$
}

1. Department of Psychology, Faculty of Social Sciences, Razi University, Kermanshah, Iran.

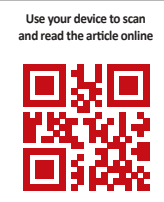

Crtation: Momeni Kh, Rafiee Z. [Relationship Between Social Support and Religious Considering With Life Satisfaction in Elderly (Persian)]. Iranian Journal of Ageing. 2018; 13(1):50-61. https://doi.org/10.21859/SIJA.13.1.50

Received: 15 Jul. 2017 Accepted: 03 Dec. 2017

Key words:

The elderly, Religious orientation, Social support, Life satisfaction

\begin{abstract}
A B S TRACT
Objectives Population is an ever-increasing phenomenon. During old age, the declining social relationships impact the lifestyle and life satisfaction. The present study investigated the correlation between social support/ religious orientation and life satisfaction among the elderly.

Methods \& Materials The present study was descriptive and correlational. The subjects included Kermanshah elderly nursing home residents in 2016. The cohort comprised of 126 elderlies (mean 69.76 and standard deviation 9), who were chosen by convenience sampling. The instruments used in this study included the social support appraisal scale, religious orientation scale, and the satisfaction with life scale. Pearson's correlation coefficient and regression and the Statistical Package for Social Science (SPSS) were used for data analysis. Results A significant correlation was established between religious orientation and life satisfaction $(P<0.05)$. Also, a correlation was observed between external religious orientation and life satisfaction 0.077 , while that between internal religious orientation and life satisfaction was $0.249 \quad(P<0.05)$. The correlation coefficient between the internal religious orientation and social support was 0.708 , while that between the external religious orientation and social support was $0.374(\mathrm{P}<0.05)$. However, no correlation was established between social support and life satisfaction. Also, internal religious orientation, external religious orientation, and social support could predict the variance of life satisfaction $\left(R^{2}=11 \%\right)$.

Conclusion Considering the need of the elderly to be supported by their relatives, the increase in social support and adherence to religious orientation could efficiently improve their life satisfaction.
\end{abstract}

\section{Extended Abstract}

\section{Objective}

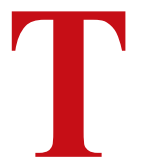

he increasing population of elderly in Iran has drawn attention to the new issues related to them; for example, social support. It is a social network providing psychological and tangible resources for individuals to be able to deal with stressful living conditions and daily issues. As the old age disabilities become common with increasing age and occasionally inevitable dependence, the role of social support in such circumstances is highlighted in improving the life satisfaction in the elderly. One of the interpersonal aspects effective on mental health is religion. Successful aging is a good model of aging in connection with the religious orientation [2]. Sine social dimension of health is the most controversial aspect of health, the current study aimed to investigate the relationship between social support/religious orientation and life satisfaction in the elderly.

* Corresponding Author:

Zahra Rafiee, MSc.

Address: Department of Psychology, Faculty of Social Science, Razi University, Kermanshah, Iran.

Tel: +98 (938) 9615930

E-mail: zahra.r3606@gmail.com 


\section{Methods and Materials}

The present study is applied research and correlational study using the descriptive method. The statistical population of the present study included all individuals aged 60 years and older residents of the Kermanshah nursing home in 2016; the cohort consisted of 200 individuals. The sample size based on the Morgan table was 126 (48 males and 78 females) mean $(\mathrm{SD})=69.76(9)$, which was determined by the available sampling method. The inclusion criteria for participation in the study were as follows: informed consent to participate in the research, ability to answer questions physically and psychologically, and residence in the elderly home. On the other hand, exclusion criteria consisted of a very severe mental illness or disorder (dementia, Alzheimer's disease, schizophrenia, and severe depression) based on the medical records and experience of grief (death of close relatives) in the last 6 months. Therefore, the investigators referred to the relevant center during the weekdays and provided the subjects with questionnaires, after introduction and explanation of research objectives, providing the necessary instructions for the completion of the questionnaires, and obtaining written consent from the elderly. The current study was approved by the Razi University of Kermanshah .

The questionnaires used in this study included social support questionnaire (Philips et al.,1976) that consisted of 23 questions to measure the social support. In the study by Khabbaz et al. (2011) [32], the calculated alpha coefficient for this questionnaire was 0.74 . Social Support Questionnaire [34] and Satisfaction With Life Scale [34] were used to assess the level of individuals' satisfaction; it contained five propositions. The reliability of this questionnaire using test-retest method was 0.82 and that using Cronbach's alpha was 0.87 . The Religious Orientation Questionnaire, containing 21 questions, by Allport and Ro (1967) [27] was used to measure the internal and external religious orientations. The internal consistency of this questionnaire using Cronbach's alpha was 0.71 , and the retest reliability was 0.74 . Pearson's correlation coefficient, simultaneous regression analysis, and SPSS software were used for data analysis.

\section{Results}

Descriptive findings showed that the mean of the external religious orientation (40.40) was higher than that of the inner religious orientation (26.83) for the variable of religious orientation. On the other hand, life satisfaction with the mean of 23.50 in the subjects was higher than the average score of 20, and the mean of social support in the subjects was $18 / 48$. Therefore, the social support can be considered as excellent. In the social support components, family support component had a higher mean (6.74) than friends' support (5.93) as well as that of the others (5.81).

These results indicated a significant relationship between one of the components of religious orientation and life satisfaction $(\mathrm{P}<0.05)$. In other words, the correlation coefficient was 0.249 between internal orientations and life satisfaction; since the correlation coefficient was positive, it can be speculated that the life satisfaction was raised by increasing the inner religious orientation. However, no significant relationship was established between the external religious orientations and life satisfaction. The correlation coefficient between these two variables was 0.77 . In addition, the results also showed a significant positive correlation between the religious orientation and social support $(0.708, \mathrm{P}<0.05)$. Thus, the social support in subjects was increased with increasing religious orientation. Furthermore, the relationship between the external religious orientation and social support was 0.374 at $\mathrm{P}<0.05$ level, which led to the conclusion that social support in subjects was increased with increasing religious orientation. However, no significant relationship was observed between social support and life satisfaction.

The results of regression test showed that inner religious orientation, external religious orientation, and social support could predict the variability of life satisfaction. $\mathrm{R}^{2}=0.11$ potentiated that these variables could significantly predict $11 \%$ of the changes in life satisfaction. In the coefficient equation, it was found that $\mathrm{B}=0.562$ as obtained in the internal religious orientation variable; this phenomenon showed that 1 unit increase in the internal religious orientation variable led to 0.562 unit increase in the life satisfaction variable. In social support, $\mathrm{B}=0.546$ showed that with 1 unit increase in social support, life satisfaction increased by 0.546 .

\section{Conclusion}

Due to the experiences of life, elderly are part of the capital of a community, and therefore, addressing their mental and physical needs is essential for the society. However, paying attention to the growth of life satisfaction of the elderly firstly requires investigating the relationship between religious orientation and social support. The results of the present study showed that religious orientation and social support were able to 
predict life satisfaction. Therefore, for attaining life satisfaction, increasing the life satisfaction of the elderly could be optimally achieved by holding group religious and fun ceremonies, as participation in such activities has been recognized as one of the sources of social support, in addition to providing spiritual dimension. Thus, altogether, the effective steps can be taken by increasing the adherence of the elderly to religious orientation and providing space for religious ceremonies in order to improve their satisfaction with life.

\section{Acknowledgments}

This research did not receive any specific grant from funding agencies in the public, commercial, or not-forprofit sectors.

\section{Conflict of Interest}

The authors declared no conflicts of interest. 


\title{
رابطه حمايت اجتماعى و جهت تيرى مذهبى با رضايت از زندتى در سالمندان
}

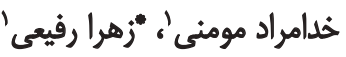

ا - كروه روانشئاسى، دانشكده علوم اجتماعى و تربيتى، دانشكاه رازي، كرمانشاه، ايران.

\begin{abstract}
حكיe

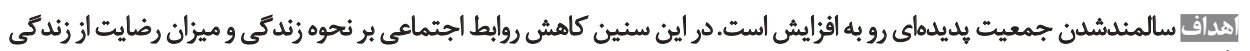

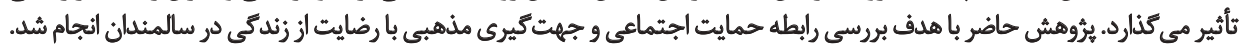

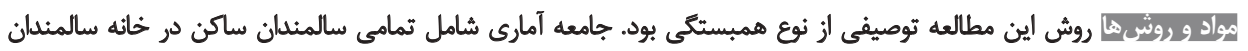

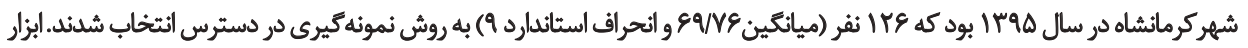

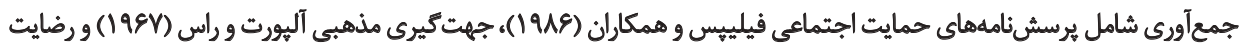

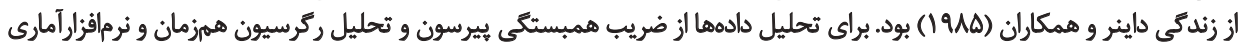
SPSS

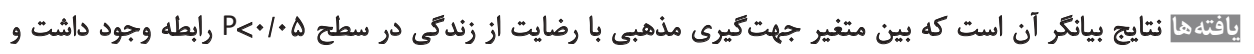

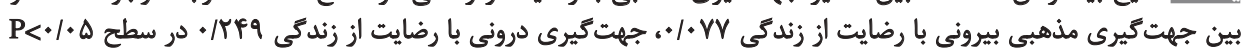

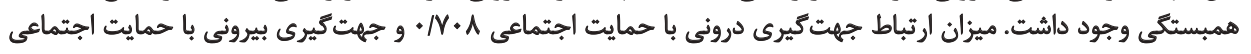
جهF

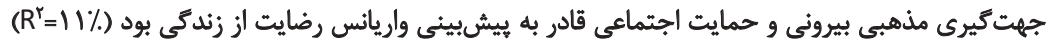

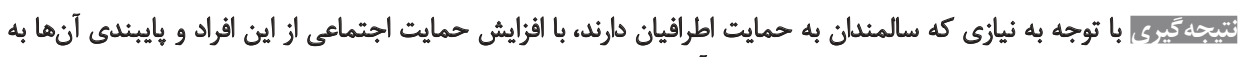

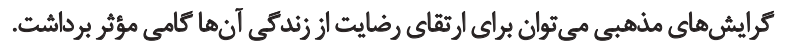

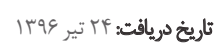

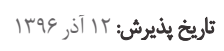

رضايت از زندكى منعكس كننده توازن بين آرزوهاى فرد و

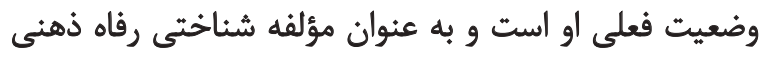

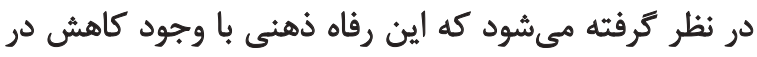

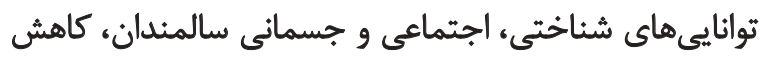

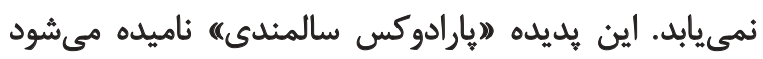

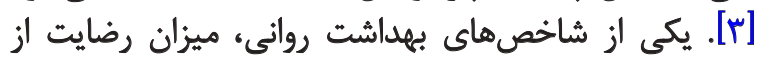

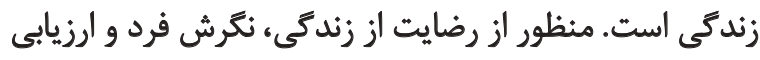

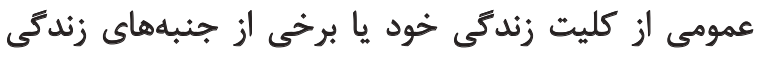

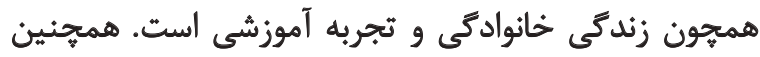

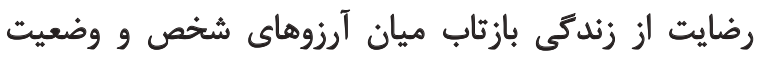

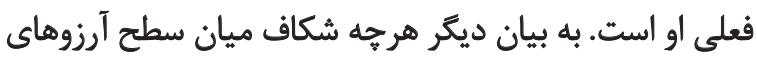

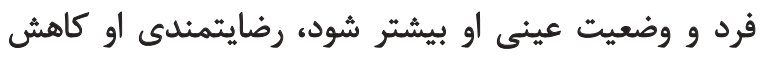

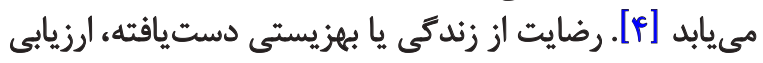

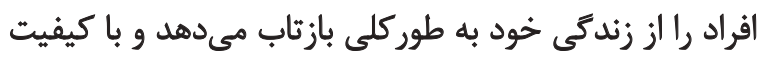

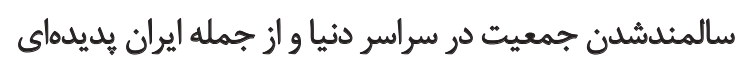

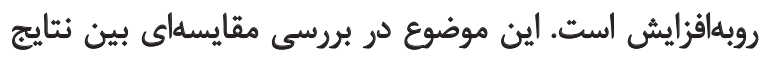

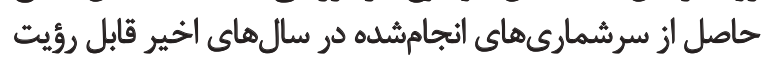

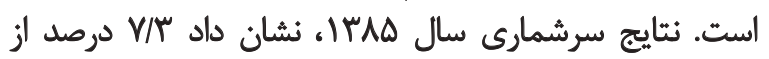

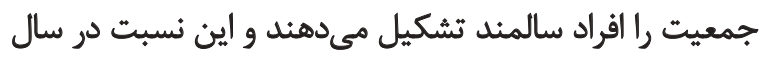

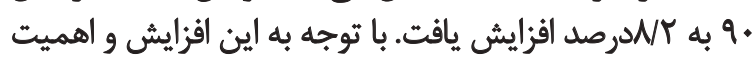

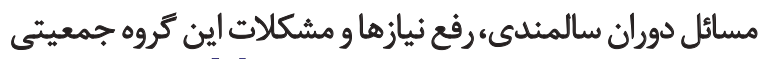
به عنوان ضرورت اجتماعى قابل طرح است [1]

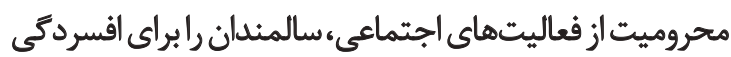

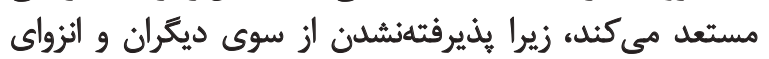

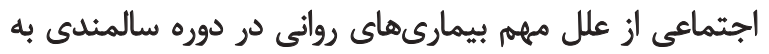

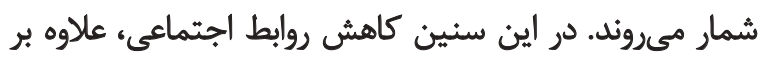

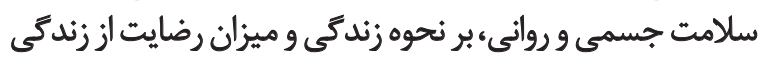

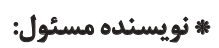

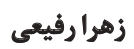

نشاني: كرمانشاهء دانشعاه رازي، دانشكده علوم اجتماعى، كروه روانشئاسى،

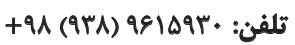
يست الكترونيكي: zahra.r3606@gmail.com 
مشخص كَزارش كردهاند سطوح بالاتر از تعاملات مذهبيى،

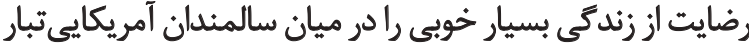

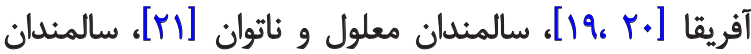

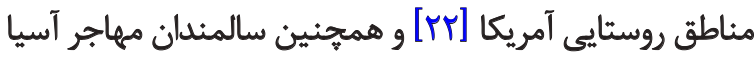

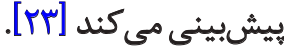

يس إز بازنشستكى، منابع فرد (امكانات مادى و منابع معنوى)

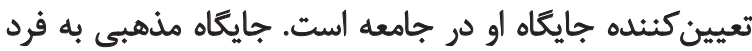

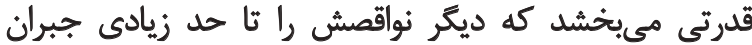

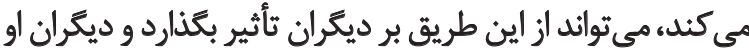

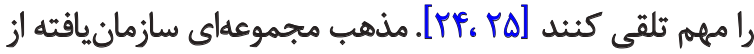

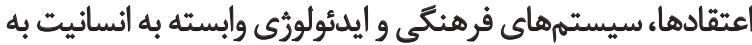

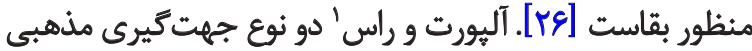

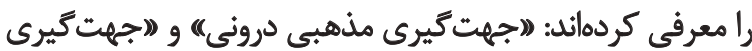

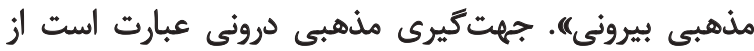

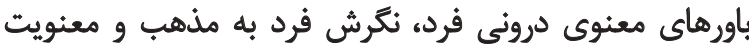

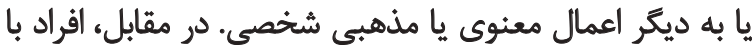

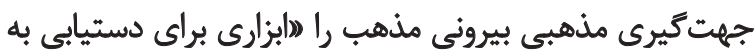

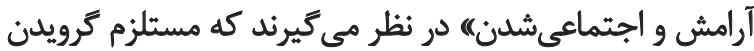

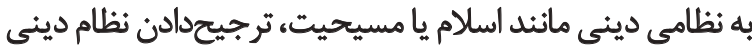

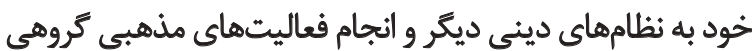

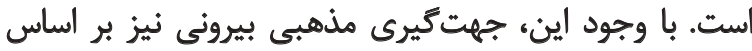

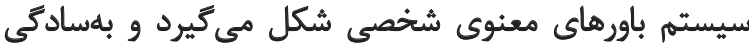

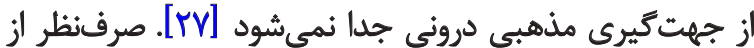

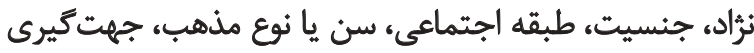
مذهبى از طريق كاركردهاى فردى و اجتماعى مختلف هميجون

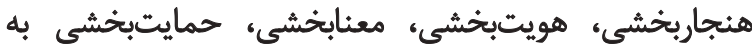

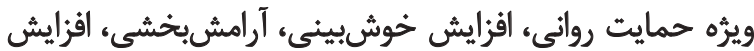

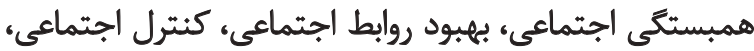

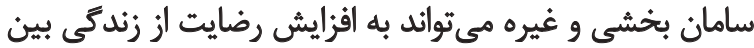

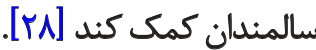

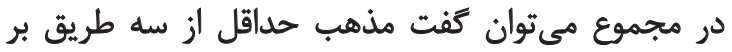

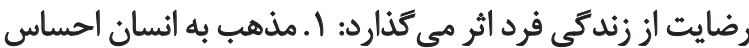

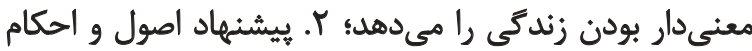

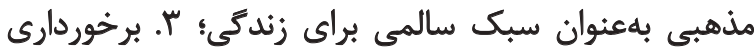

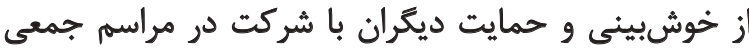

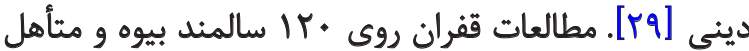

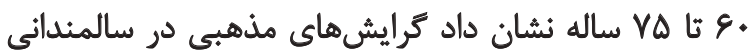

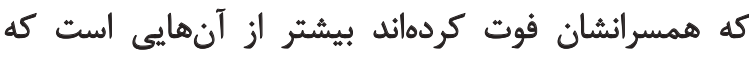

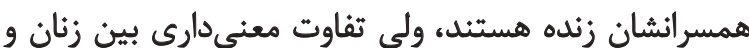

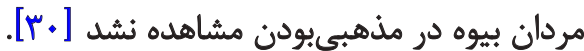
از آنجا كه يديده سالمندى در تمامى جنبههاى زندكى

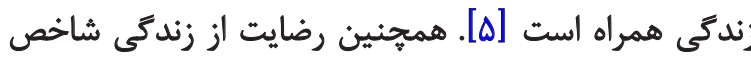

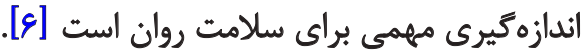
يكى از متغيرهايى كه بر ميزان رضايت از زندكى مؤثر است،

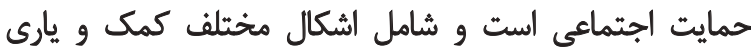

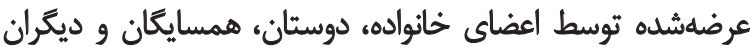

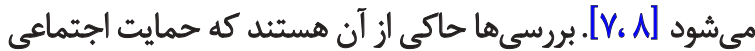

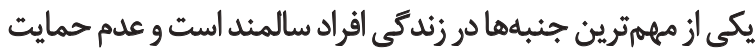

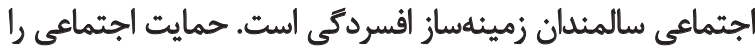

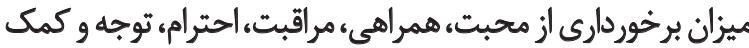

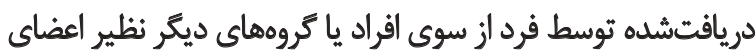

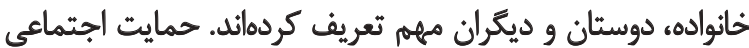

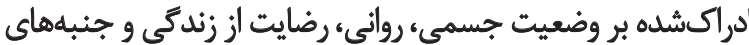

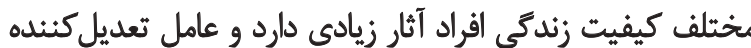

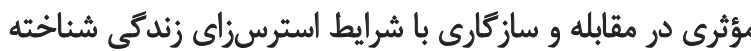

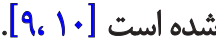

تحقيقات انجامشده در جيند دها كذشته، در ميان طيف

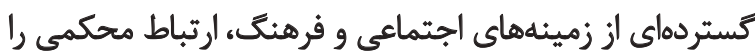

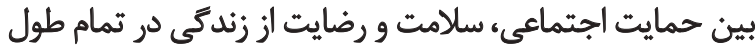

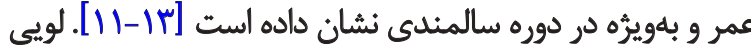

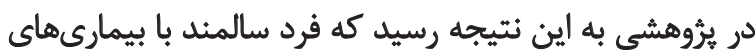

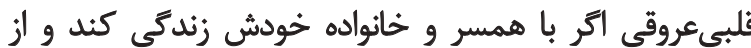

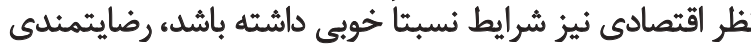

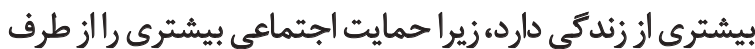

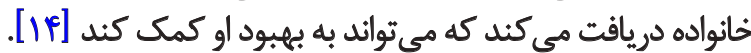

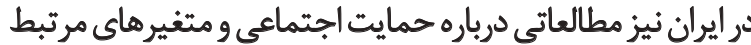

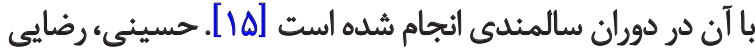

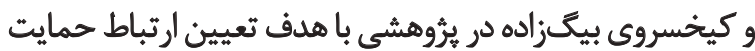

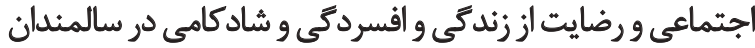

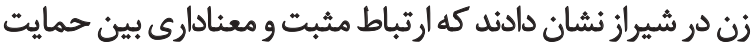

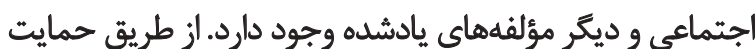

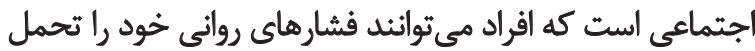

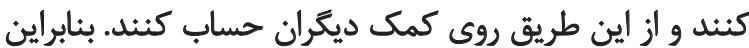

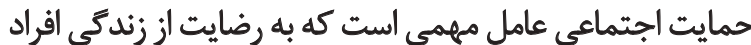

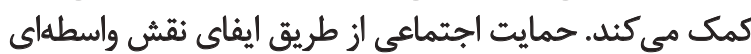

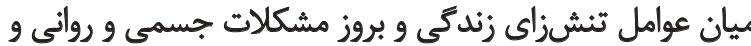

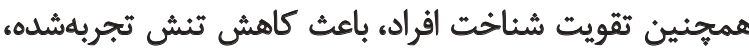

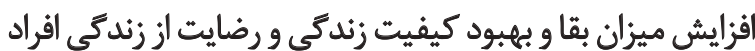

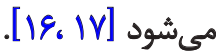

متغير مهم تأثير كذار ديكر بر رضايت از زندكى سالمندان،

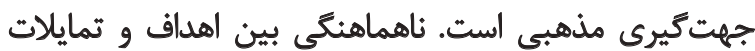

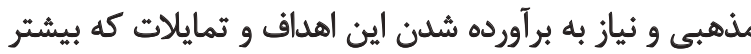

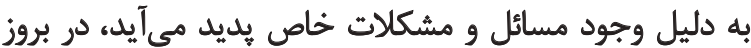

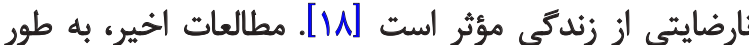


هبرسشنامه حمايت اجتماعى

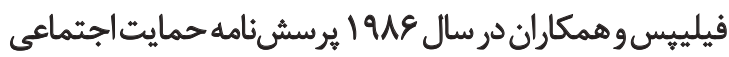

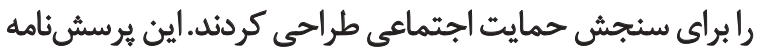

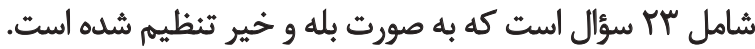

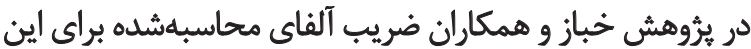

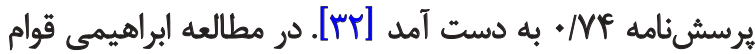

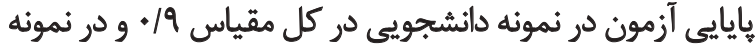

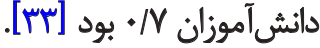

$$
\text { مثياس رضايث الز زثندئى }
$$

مقياس رضايت از زندكى داينر و همكاران براى ارزيابي ميزان

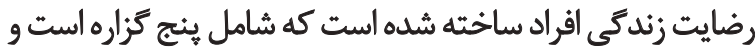

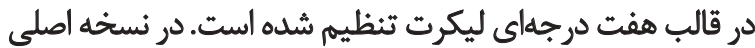

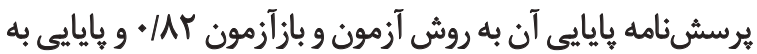

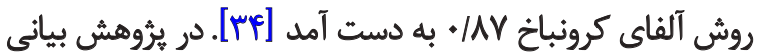

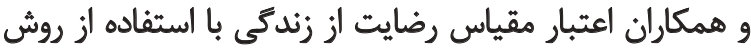

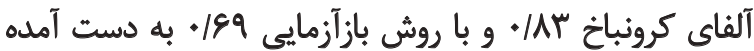

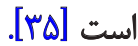

\section{مقياس جهت تميرى مذهبي}

آليورت و راس در سال 199V مقياس جهت ديرى مذهبى را

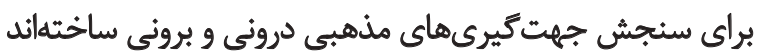

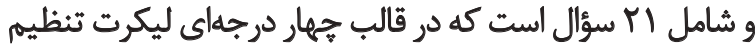

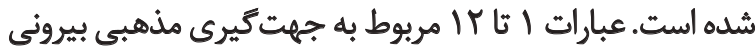

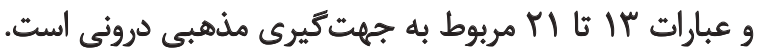

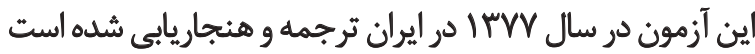

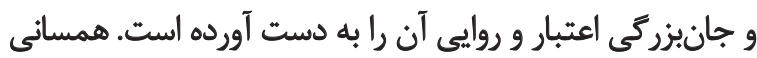

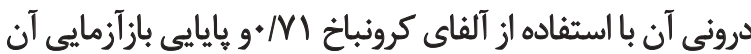

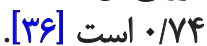

يس إز گرفتن مجوز از معاونت ثرؤهشى بهزيستى استان

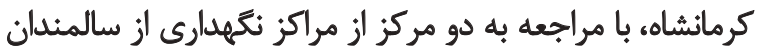

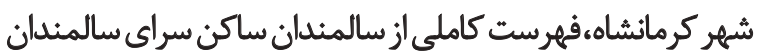

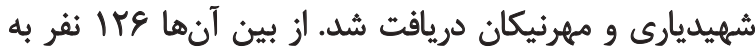

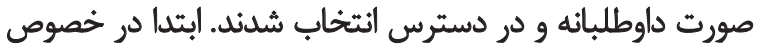

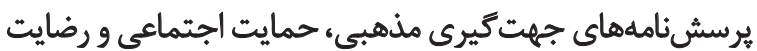

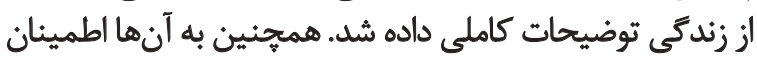

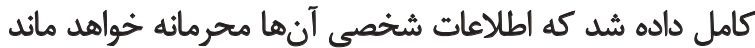

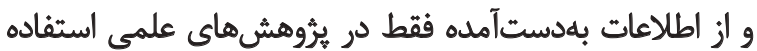

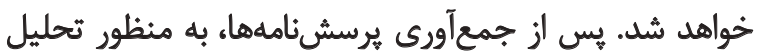

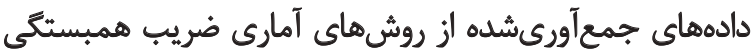

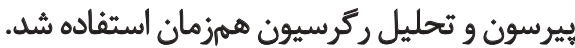

جوامع بشرى، از جمله در طيف گستردهاى از ساختارهاى

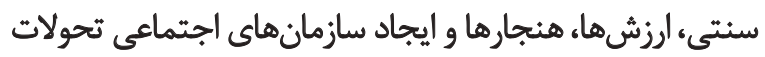

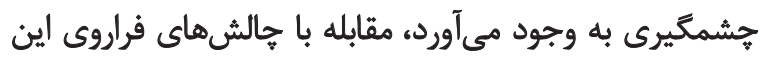

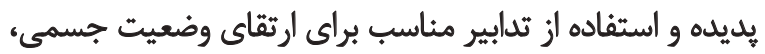

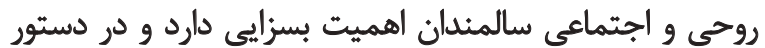

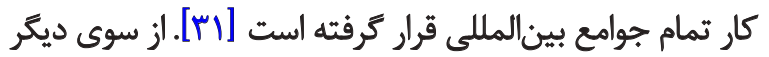

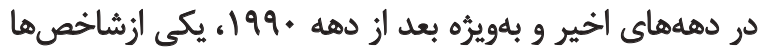

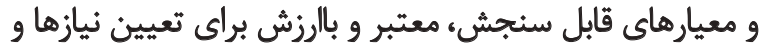

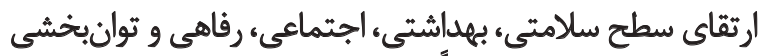

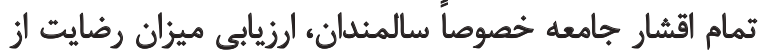
زندكى آنهاست.

بنابراين با توجه به آنجه كفته شد، اهميت دوره سالمندى

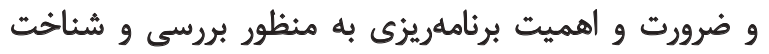

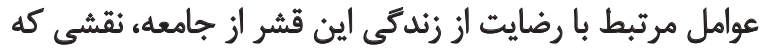

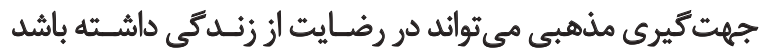

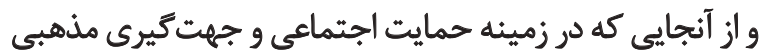

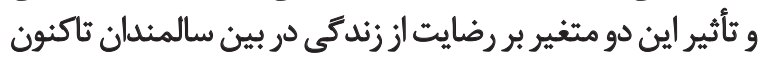

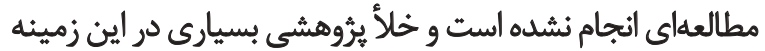

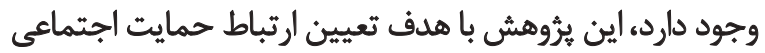

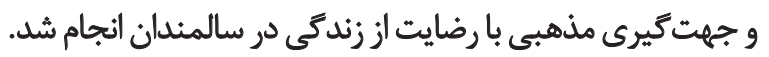

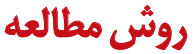

روش ثرؤش حاضر، توصيفى از نوع همبستخى است. جامعه

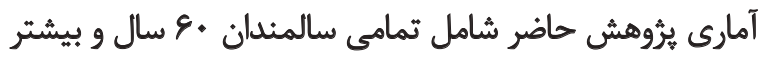

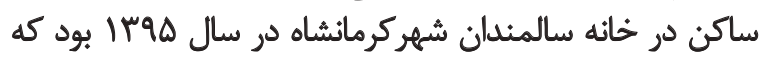

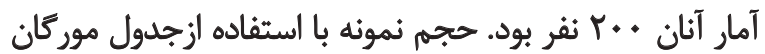

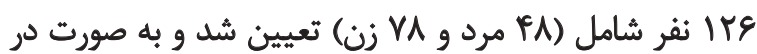

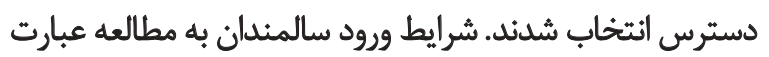

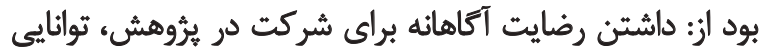

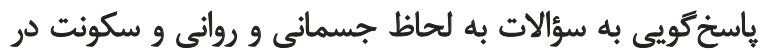

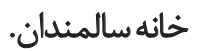

معيارهاى خروج از مطالعه نيز شامل وجود بيمارى يا اختلال

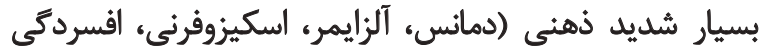

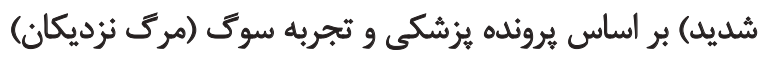

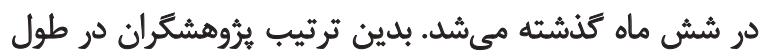

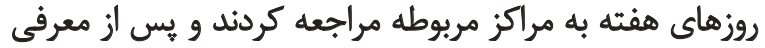

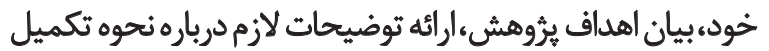

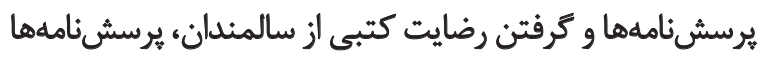

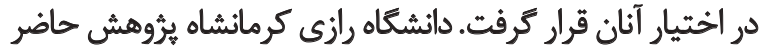

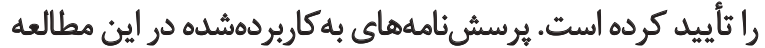

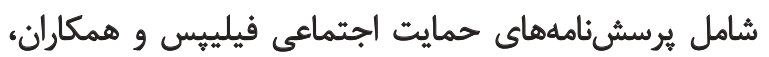

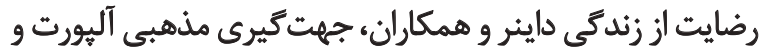
راس و بركة اطلاعات جمعيتشناختى بوني 


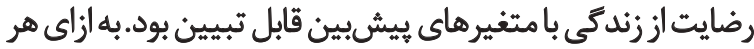

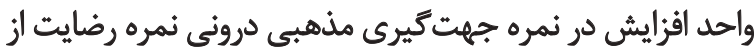

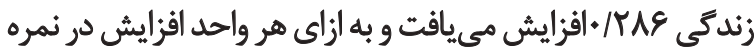

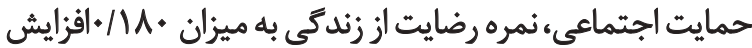

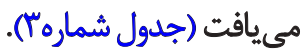

بحث

اين مطالعه باهدف بررسى رابطه حمايت اجتماعى وجهت زئيرى

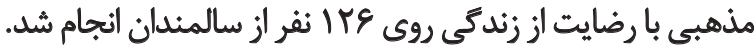

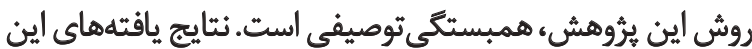

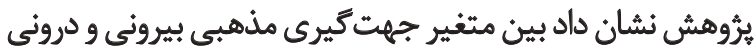

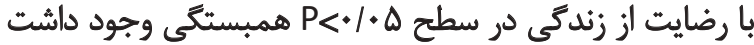

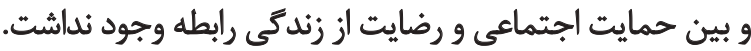

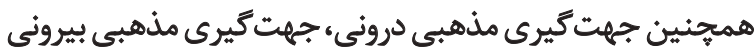

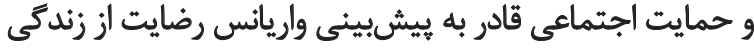

$$
\text { بود (R) }
$$

مطالعه مبنى بر اينكه بين جهت كيرى مذهبى با رضايت

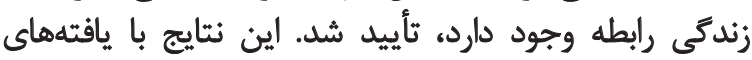

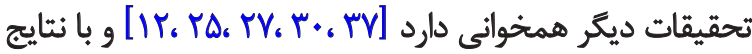

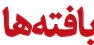

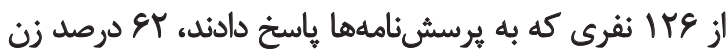

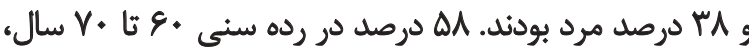

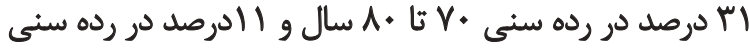

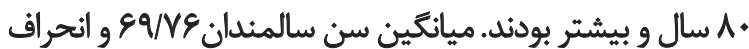

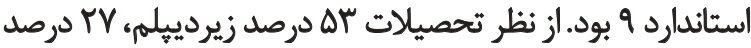

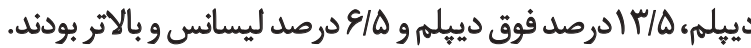

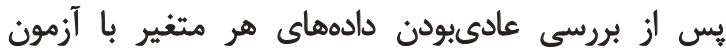

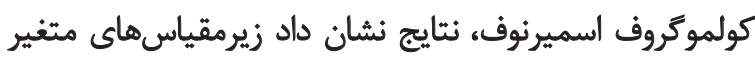

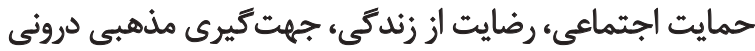

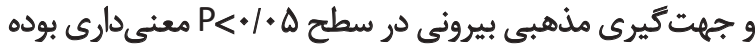

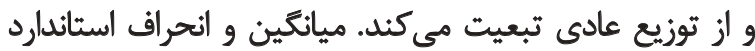
متغيرهاى يُؤهش در جدول شماره إكزارش شده است.

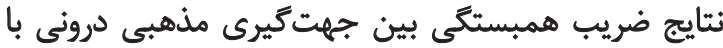

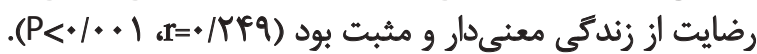

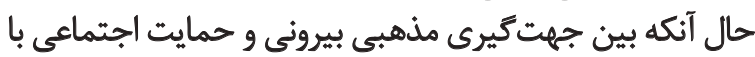

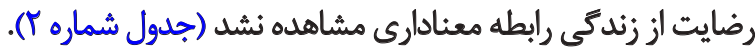
نتايج ضريب ركرسيون نشان داد كه لا درصد از واريانس

\begin{tabular}{|c|c|c|}
\hline انحراف استاندارد & مياتكين & مثغير \\
\hline q/Prf & $f \cdot / f \cdot$ & جهت Sيرى مذهبى بيرونى \\
\hline r/AEY & rg/Ar & جهت كيرى مذهبى درونى \\
\hline$V / . . q$ & $\pi / \Delta$ & رضايت از زندكى \\
\hline I/T"Ar & $g / M^{e}$ & حمايت خانواده \\
\hline $1 / 4 V$ & ه/q & حمايت دوستان \\
\hline W/Tr & $\Delta / A)$ & حمايت ديكران \\
\hline$r / r \cdot F$ & WFA & حمايت الجتماعى \\
\hline
\end{tabular}
جدول ا. ميانكين و انحراف استاندارد متغيرهاى بُؤهش

\begin{tabular}{|c|c|c|c|}
\hline جهت كيرى مذهبي درونى & جهت كيرى مذهبي ييروني & رضايت از زندكّى & متغير \\
\hline & &.$/ \cdot W$ & جهت كيرى هذهبى بيرونى \\
\hline & $\cdot / r+T^{\prime *}$ & $. / M \times q * *$ & جهت كيرى مذهبى درونى \\
\hline$* N+1$ &.$/ m r^{\circ}$ &.$/ \triangle A A$ & حمايت اجتماعي \\
\hline
\end{tabular}

جدول Y. ماتريس همبستكي متغيرهاي جهت كيرى مذهبي، حمايت اجتماعي و رضايت از زندكى 
جدول ؟. يُيشبينى رضايت از زندكى با جهت كيرى مذهبى بيرونى، جهت كيرى مذهبى درونى و حمايت اجتماعى

\begin{tabular}{|c|c|c|c|c|c|c|c|c|}
\hline معنى سطرى & t آهاره t & الميثانيدارود & استاندارود & برآورد اوليه & آماره فيشر & تعيبن & ضريب ركرسيون & \\
\hline$* *+)^{*}$ & & & & & $\Delta / * 19$ & .1111 & ת זM/ & رضايت از زندكى \\
\hline TH & $-\cdot N N E$ & & V/A)\& & - g/IFT & & & & ثابت مدل \\
\hline .1 .9 & $\mathbb{N} \cdot A$ &.$/ 10$ & .1 .90 & .1111 & & & & جهت كيرى هذهبى بيرونى \\
\hline $.1 . .1 *$ & T/TVO & - KN &.$/ 1 k T$ & . IDST & & & & جهت Sيرى مذهبي درونى \\
\hline$\because \cdot \mathrm{HA}^{*}$ & $r / . Q$ &.$/ M$ &. $\mid r e I$ & . lape & & & & حمايت اجتماعي \\
\hline
\end{tabular}

سال هاى عمر خود را با احساس ارزشمندى و مؤثر بودن بخذراند.

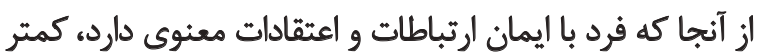

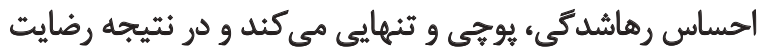

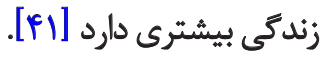

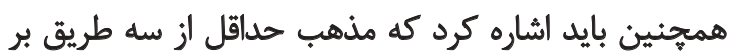

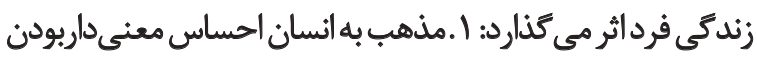

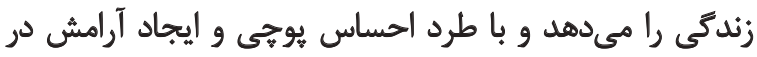

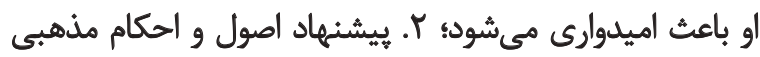

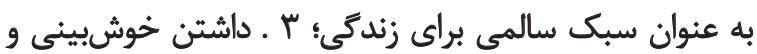

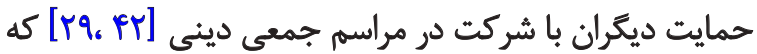

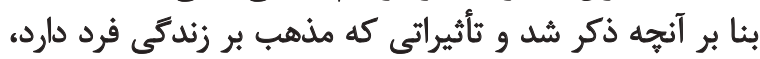

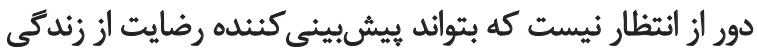

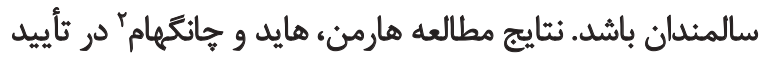

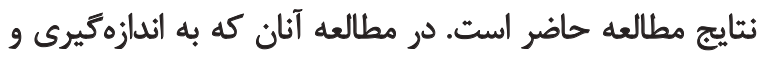

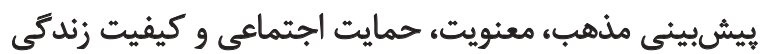

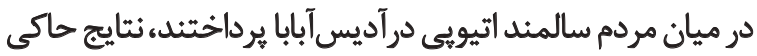

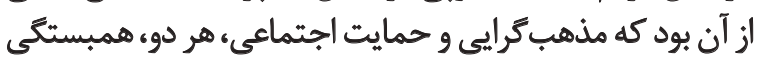

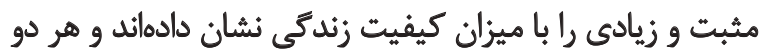

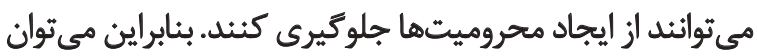

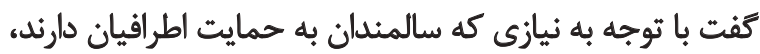

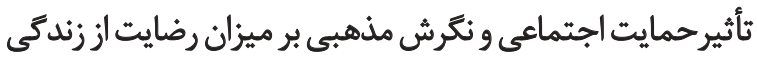

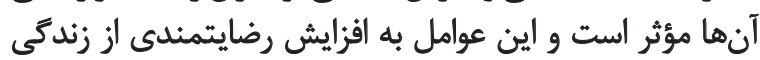
آن آنها كمك مؤر است كند [ين

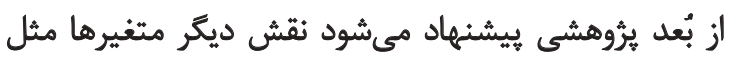

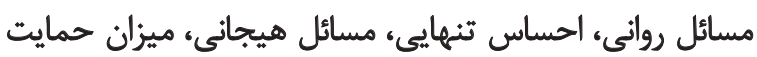

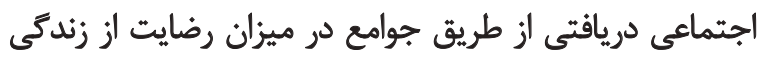

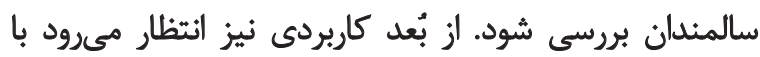

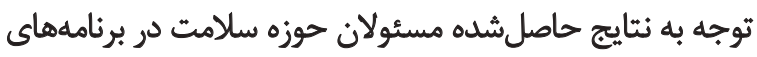

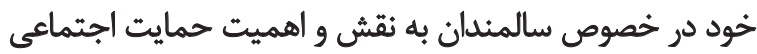

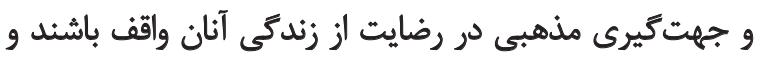
تداركات و اقدامات مقتضي رئ را انجام دهند رضي.

2. Hamren, Hyde, Chungkham

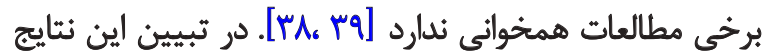

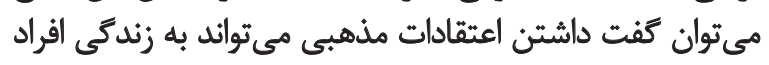

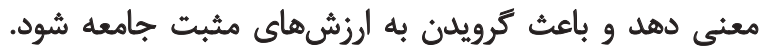

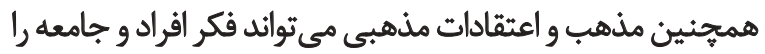

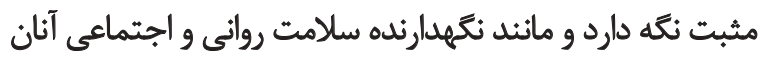

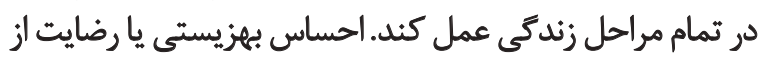

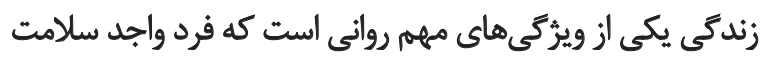

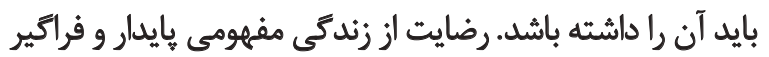

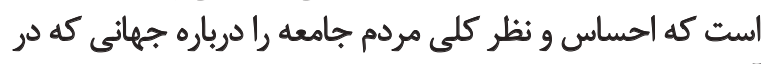

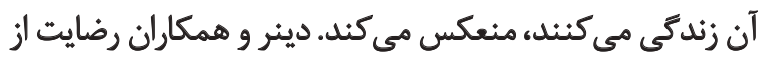

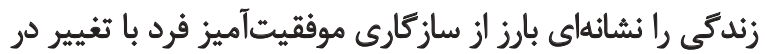

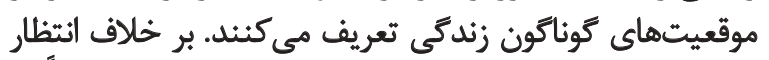

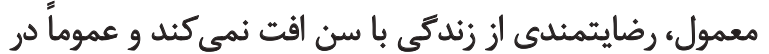

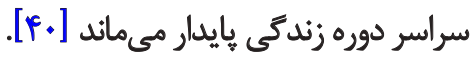

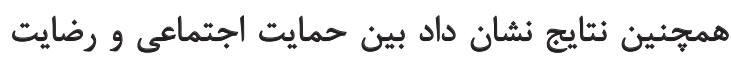

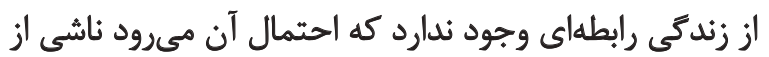

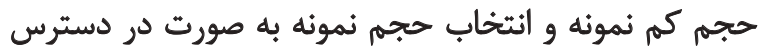

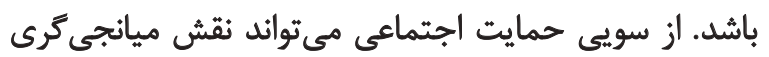

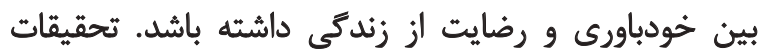

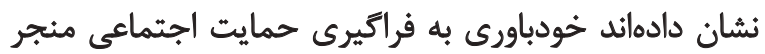

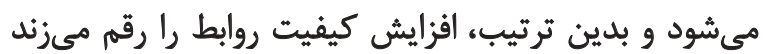

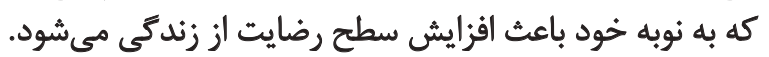

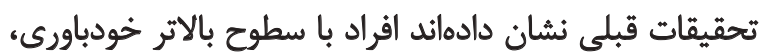

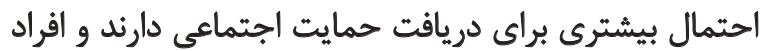

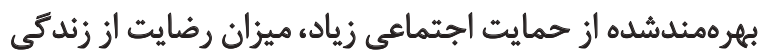

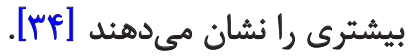

نتايج حاكى از آن بود كه جهت كيرى مذهيبى و حمايت

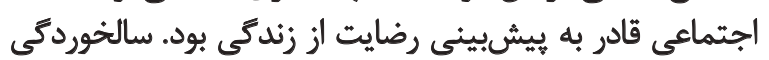

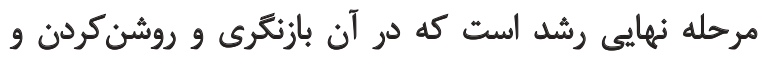

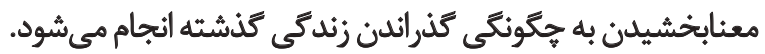

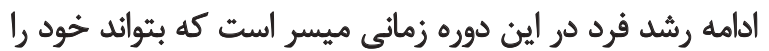

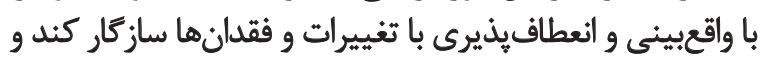




\section{نتيجه}

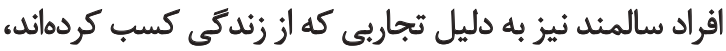

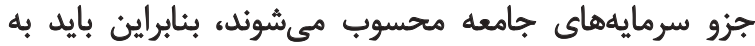

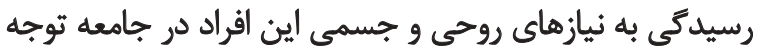

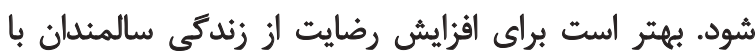

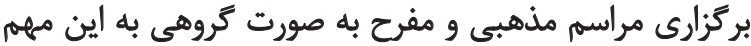

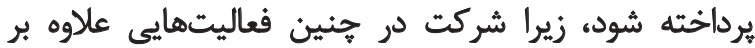

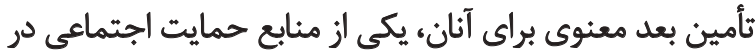

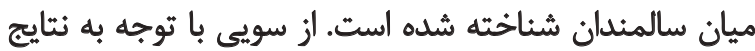

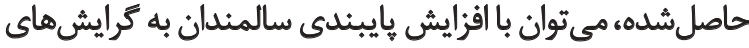

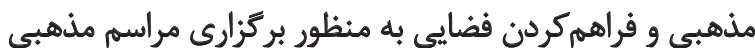

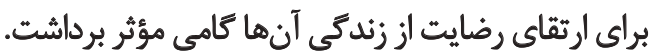

از جمله محدوديتهايى كه در اين ثئوهش وجود داشت بردي،

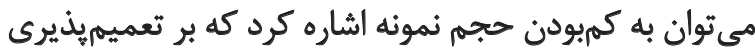

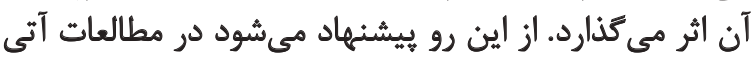

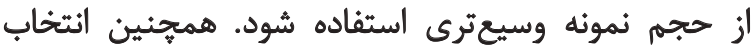

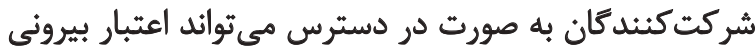

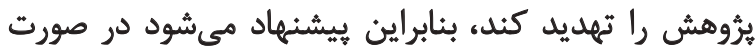

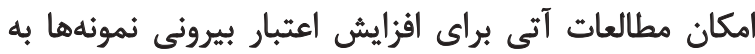

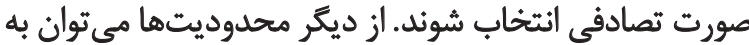

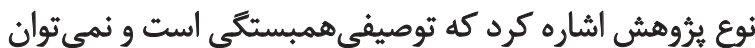

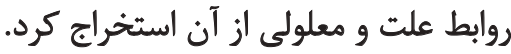

$$
\text { تشكير و قدرواني تئي }
$$

از تمامى سالمندان ساكن در سراى سالمندان شهركرمانشاه كه

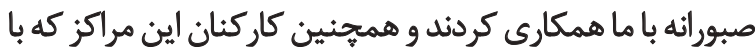

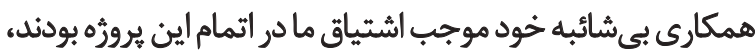

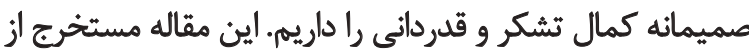
بإيان إمه نيست و حامى مالى ندارد. 


\section{References}

[1] World Health Organization. Ageing and life course. Geneva: World Health Organization; 2015.

[2] Hojati H, Hojati H, Sharifnia SH, Salmasi A, Hoseinzadeh A, Farhadi S. [Comparison of loneliness, belonging and life satisfaction in the elderlies living at home with the nursing home residents (Persian)]. Journal of Health \& Care. 2012; 14(3):9-15.

[3] Nakahara J. Effects of social activities outside the home on life satisfaction among elderly people living alone. International Journal of Psychological Studies. 2013; 5(1):112-20. doi: 10.5539/ijps. v5n1p112

[4] Shamsaei F, Oshtorani F, Oshtorani A. [Comparison of life satisfaction in blind and deaf students with normal students of Hamadan's schools in 2014 (Persian)]. Pajouhan Scientific Journal. 2014 13(1):52-60.

[5] Lu M, Yang G, Skora E, Wang G, Cai Y, Sun Q, et al. Self-esteem, social support,and life satisfaction in Chineseparents of children with autism spectrum disorder. Research in Autism Spectrum Disorders. 2015; 17:70-77. doi: 10.1016/j.rasd.2015.05.003

[6] Pilar Matud M, Bethencourt JM, Ibáñez I. Relevance of genderroles in life satisfaction in adult people. Personality and Individual Differences. 70: 206-211. doi: 10.1016/j.paid.2014.06.046

[7] Barrera M, Sandler IN, Ramsay TB. Preliminary development of a scale of social support: Studies on college students. American Journal of Community Psychology. 1981; 9(4):435-47. doi: $10.1007 /$ bf00918174

[8] Hamren K, Chungkham HS, Hyde M. Religion, spirituality, social support and quality of life: Measurement and predictors CASP-12(v2) amongst older Ethiopians living in Addis Ababa. Aging \& Mental Health. 2014; 19(7):610-21. doi: 10.1080/13607863.2014.952709

[9] Ingersoll B, Silverman A. Comparative group psychotherapy for the Aged. The Gerontologist. 1978; 18(2):201-6. doi: 10.1093/geront/18.2.201

[10] Farhadi A, Obeidavi Z, Movahedi Y, Rahimi M, Mobasher A. [The comparison and relationship of social support and stress with life satisfaction among the elderly of Khorramabad city in 2013 (Persian)]. yafte. 2015; 17(2):14-22

[11] Crimmins EM, Preston SH, Cohen B. The role of social networks and social integration. In: Crimmins EM, Preston SH, Cohen B, editors. Explaining divergent levels of longevity in high-income countries. Washington, D.C.: National Academies Press; 2011.

[12] Muckenhuber J, Stronegger WJ, Freidl W. Social capital affects the health of older people more strongly than that of younger people. Ageing and Society. 2012; 33(5):853-70. doi: 10.1017/ s0144686x12000219

[13] Lee EO. Religion and spirituality as predictors of wellbeing among Chinese American and Korean American older adults. Journal of Religion, Spirituality \& Aging. 2007; 19(3):77-100. doi: 10.1300/j496v19n03_06

[14] Fardinejad Z, Ahadi H. [The relationship of general health and perceived social support with life satisfaction in elderly people (Persian)]. Journal of Behavioral Sciences. 2013; 5(15):87-100.

[15] Hoseini SM, Rezaei A, Keykhosravi Z. [A comparison of old men and women's social support, life satisfaction, happiness and depression (Persian)]. Sociology of Women. 2011; 2(4):143-61.
[16] Ghaedi GH, Yaaghoobi H. [A study on the relationship between different dimensions of perceived social support and different aspects of wellbeing (Persian)]. Armaghan-e- Danesh. 2008; 13(2):69-81.

[17] Parpoochi B, Ahmadi M, Sohrabi F. [The relationship of religiousness and social support with life satisfaction among university students (Persian)]. Knowledge \& Research in Applied Psychology. 2013; 14(3):60-68

[18] Masoudi R, Soleimani MA, Qorbani M, Hasheminia AM, Pour Dehkordi AH, Bahrami N. [The effect of family centered empowerment model on the quality of life in elderly people (Persian)]. Journal of Qazvin University of Medical Sciences. 2010; 1(14):51-64.

[19] Krause N. Common facets of religion, unique facets of religion, and life satisfaction among older African Americans. The Journals of Gerontology Series B: Psychological Sciences and Social Sciences. 2004; 59(2):S109-17. doi: 10.1093/geronb/59.2.s109

[20] Levin JS, Chatters LM, Taylor RJ. Religious effects on health status and life satisfaction among black Americans. The Journals of Gerontology Series B: Psychological Sciences and Social Sciences. 1995; 50B(3):S154-63. doi: 10.1093/geronb/50b.3.s154

[21] Moberg DO. Disabilities, spirituality, and well-being inlate life: Research foundations for study and practice. Journal of Religion, Spirituality \& Aging. 2008; 20(4):313-40. doi: $10.1080 / 15528030802232379$

[22] Yoon DP, Lee EKO. The impact of religiousness,spirituality, and social support on psychological well-being among older adults in rural areas. Journal of Gerontological Social Work. 2007; 48(3-4):281-98. doi: 10.1300/j083v48n03_01

[23] Roh S, Lee KH, Yoon DP. General well-being of Korean immigrant elders: The significance of religiousness/spirituality and social support. Journal of Social Service Research. 2013;39(4):483-97. doi: 10.1080/01488376.2012.709451

[24] Hazan H. Antropology of aging. In: Bergman S, Margulec I, editors. Aging in Israel: Selected topics in gerontology. Tel Aviv: Publishing House; 1984.

[25] Zahednejhad SH, Fazeli Y, Rezayi H, Haghighizadeh MH. [Association of coping strategies and religiosity with life satisfaction of old people living in nursing homes of Ahvaz city, Iran. (Persian)]. Salmand. 2016; 10(4):30-39.

[26] Scholer K. The everything world's religions Book: Explore the beliefs, traditions, and cultures of ancient and modern religions. Avon, Massachusetts: Adams Media; 2010.

[27] Park J, Roh S, Yeo Y. Religiosity, social support, and life satisfaction among elderly Korean immigrants. Gerontologist. 2012; 52(5):641-9. doi: 10.1093/geront/gnr103

[28] Hajizade Meimandi M, Zare Shahabadi A, Marvinam N, Abotorabi Zarchi F. [A survey of the relationship between religiosity and life satisfaction among elderly women in Yazd (Persian)]. Womens Strategic Studies. 2012; 15(58):191-235.

[29] Rabani R, Behesgti SS. [An empirical study of the relationship between religiosity and life satisfaction (Persian)]. Oloom-e Ejtemaei. 2011; 8(1):79-102.

[30] Aliakbari Dehkordi M, Oraki M, Barghi Irani Z. [Relation betweereligious orientation with anxiety about death, and alienation in aged peoples (seniors) in Tehran (Persian)]. Social Psychology Research. 2011; 1(2):140-59. 
[31] Amani R. [The effectiveness of structured group reminiscence on the enhancement of elderly's positive self-concept (Persian)]. Journal of Aging Psychology 2015; 1(1):1-9.

[32] Khabaz M, Behjati Z, Naseri M. [Relationship between social support and coping styles and resiliency in adolescents (Persian)]. Journal of Applied Psychology 2011; 5(4):108-123.

[33] Ebrahimighavam S. [Check the validity of the three concepts of locus of control, self-esteem and social support (Persian)] [MSc. thesis]. Tehran: Islamic Azad University; 1992.

[34] Diener E, Emmons RA, Larsen RJ, Griffin S. The satisfaction with life scale. Journal of Personality Assessment. 1985; 49(1):715. doi: 10.1207/s15327752jpa4901_13

[35] Bayani A, Mohammad Koocheky A, Goodarzi H. [The reliability and validity of the satisfaction with life scale (Persian)]. Developmental Psychology. 2007; 3(11):259-265.

[36] John Bozorgi, M. [Evaluate the effectiveness of psychotherapy with or without religious orientation of the anxiety and stress. (Persian)] [PhD thesis]. Tehran: University of Tarbiat Modaress; 1998.

[37] Shkolnik T, Weiner C, Malik L, Festinger Y. The effect of Jewish religiosity of elderly Israelis on their life satisfaction, health, function and activity. Journal of Cross-Cultural Gerontology. 2001; 16(3):201-19. doi: 10.1023/a:1011917825551

[38] Van Ness PH, Kasl SV. Religion and cognitive dysfunction in an elderly cohort. The Journals of Gerontology Series B: Psychological Sciences and Social Sciences. 2003; 58(1):S21-S29. doi: 10.1093/geronb/58.1.s21

[39] Chen YY, Koenig HG. Do people turn to religion in times of stress? An examination of change in religiousness among elderly, medically ill patients. Journal of Nervous and Mental Disease. 2006; 194(2):114-20. doi: 10.1097/01.nmd.0000198143.63662.fb

[40] Diener E, Suh EM, Lucas RE, Smith HL. Subjective wellbeing: Three decades of progress. Psychological Bulletin. 1999; 125(2):276-302. doi: 10.1037/0033-2909.125.2.276

[41] Lotfabadi H. [Development Psychology (2) Adolescence, youth and adults (Persian)]. Tehran: SAMT; 2005.

[42] Lee EK, Sharpe T. Understanding religious/spiritual coping and support resources among African American older adults: A mixed-method approach. Journal of Religion, Spirituality \& Aging. 2007; 19(3):55-75. doi: 10.1300/j496v19n03_05 
